\section{$\underset{\substack{\text { hommes } \\ \text { \& migrations }}}{ }$}

\section{Hommes \& migrations}

Revue française de référence sur les dynamiques

migratoires

$1288 \mid 2010$

Langues et migrations

\title{
Radiographie sommaire des pratiques de l'arabe maghrébin en France
}

Alexandrine Barontini

\section{(2) OpenEdition}

\section{Journals}

Édition électronique

URL : http://journals.openedition.org/hommesmigrations/872

DOI : 10.4000/hommesmigrations.872

ISSN : 2262-3353

Éditeur

Musée national de l'histoire de l'immigration

Édition imprimée

Date de publication : 1 novembre 2010

Pagination : 104-109

ISSN : 1142-852X

Référence électronique

Alexandrine Barontini, « Radiographie sommaire des pratiques de l'arabe maghrébin en France », Hommes \& migrations [En ligne], 1288 | 2010, mis en ligne le 29 mai 2013, consulté le 20 avril 2019. URL : http://journals.openedition.org/hommesmigrations/872 ; DOI : 10.4000/

hommesmigrations.872 


\section{Radiographie sommaire des pratiques de l'arabe maghrébin en France}

Par Alexandrine Barontini, doctorante, Inalco (Paris)-Lacnad (EA 4092) /

Université de Provence Aix-Marseille 1-Iremam (UMR 6568)

L'arabe maghrébin en France est très souvent associé en premier lieu aux travailleurs immigrés d'Afrique du Nord et à leurs descendants. Or il s'agit d'une image tronquée des locuteurs de cette langue de France parlée par près de trois millions de personnes. Juifs sépharades, harkis ou pieds-noirs continuent de la pratiquer. Si la transmission de cette langue s'effectue surtout dans le cadre familial par manque de reconnaissance institutionnelle, elle véhicule un univers culturel qui a su gagner sa place dans l'espace public. 
Il y a onze ans l'arabe maghrébin était reconnu langue de France (aux côtés des langues régionales métropolitaines et d'outre-mer, mais aussi du berbère, de l'arménien occidental, du yiddish, du judéo-espagnol et du romani), suite à la signature en 1999 de la charte européenne des langues régionales et minoritaires. La reconnaissance réelle et la valorisation institutionnelle se font attendre parce que la charte n'a pas été ratifiée et ne le sera pas sans modification de la Constitution ${ }^{(1)}$.

Or la réalité de pratiques vivantes de l'arabe maghrébin en France est de plus en plus visible. Une recherche qualitative, empirique, non statistique, permet d'en relever les principaux aspects ${ }^{(2)}$.Il s'agit de dresser un tableau de ces pratiques en commençant par la description des locuteurs et des variétés employées, avant d'en venir aux pratiques proprement dites.

\section{Qu'est-ce que l'arabe maghrébin et qui le parle?}

Larabe maghrébin est une catégorisation d'ordre linguistique. Il désigne les variétés vernaculaires d'arabe parlées dans le nord de l'Afrique (de la Mauritanie jusqu'à l'ouest de l'Égypte) et au-delà (en diaspora, notamment en France); on peut également y adjoindre le maltais, langue officielle de l'île de Malte, qui, bien que ses locuteurs ne le considèrent pas comme de l'arabe, est apparenté linguistiquement à l'arabe maghrébin ${ }^{(3)}$. En France ${ }^{(4)}$, les variétés présentes sont très majoritairement celles du Maroc, d'Algérie et de Tunisie. Lorsque l'on pense à l'arabe maghrébin en France, très souvent on pense en premier lieu aux immigrés d'Afrique du Nord et à leurs descendants. Or c'est une image tronquée des locuteurs. Étudier les pratiques de l'arabe maghrébin en France suppose que l'on s'intéresse en premier lieu aux phénomènes migratoires, liés à la colonisation du nord de l'Afrique.

Ces migrations et ces implantations ont eu lieu pendant et après la colonisation. Elles ont ainsi concerné les travailleurs immigrés, bien sûr, mais également d'autres populations qui se sont installées en France pour d'autres raisons et selon d'autres modalités ; soit des juifs d'Afrique du Nord, et, parmi les personnes venues d'Algérie, des harkis et des pieds-noirs ${ }^{(5)}$. Ces derniers ne sont pas forcément tous arabophones, et, quand ils le sont, il n'y a, semble-t-il, pas de transmission linguistique directe ou consciente.

Il faut enfin ajouter les descendants de toutes ces personnes, qui ont la nationalité française et n'ont pas vécu la migration : nombre de citoyens français ont reçu cette langue en héritage de leurs parents ou grands-parents.

Enfin, ces distinctions sociohistoriques étant posées, cela ne résout pas le problème du comptage exact des locuteurs de l'arabe maghrébin. D'abord, parce que parmi les populations citées il y a un grand nombre de berbérophones, même si ceux-ci sont 
souvent aussi arabophones et même parfois transmettent l'arabe maghrébin. Ensuite, parce qu'il n'existe pas d'étude statistique complète sur ce thème des pratiques linguistiques. Cela s'explique par la loi française qui interdit tout recensement statistique selon des critères "ethniques" ou religieux, par exemple. On dispose, malgré tout, d'indications diverses ${ }^{(6)}$ qui conduisent à estimer le nombre de locuteurs autour de trois millions.

\section{Transmission et apprentissage}

L'apprentissage de l'arabe maghrébin en France se fait à travers la transmission familiale, ou du moins en contexte non institutionnel, cette langue n'étant enseignée qu'à l'université et dans de rares associations ${ }^{(7)}$. On sait, à partir des données de "l'enquête famille ${ }^{(8)}$," que "l'arabe est devenu l'héritage linguistique de $3 \%$ des adultes vivant en France ${ }^{(9)}$ " et qu'il s'agit de la langue de l'immigration la plus transmise ${ }^{(10)}$. Si la médiatisation et l'emploi de l'arabe maghrébin dans la création artistique française sont indéniables ${ }^{(11)}$, la reconnaissance scolaire et institutionnelle est largement à la traîne. La transmission linguistique familiale est un phénomène complexe, interactif, qui ne s'effectue pas à sens unique, puisqu'il peut être le résultat d'une demande, d'une volonté de (ré)appropriation de la part des enfants. Il y a donc bien une dimension d'acquisition ${ }^{(12)}$, et ce, à plusieurs niveaux : par le biais intergénérationnel, mais également par le biais des amis, des voisins, etc. À titre d'exemple, un de mes informateurs ${ }^{(13)}$ a pu développer sa pratique de l'arabe marocain grâce à la fréquentation d'une synagogue, en région parisienne, où les fidèles, en majorité originaires du Maroc, emploient cette langue pour plaisanter, notamment. Cet emploi de l'arabe marocain vient ainsi renforcer la convivialité entre eux. Pour une autre de mes informatrices ${ }^{(14)}$, l'affirmation de sa pratique de l'arabe marocain s'est effectuée grâce à son compagnon, qui a grandi au Maroc.

Les modalités de cette interaction sont donc propres à chacun et il n'est pas pertinent d'établir des modèles de transmission ${ }^{(15)}$. Il est toutefois évident qu'une langue se transmet mieux lorsqu'elle bénéficie d'une valorisation au sein de la famille, mais aussi à l'extérieur de celle-ci. D'autant plus que la suprématie du français, de l'idéologie monolingue ${ }^{(16)}$, mêlée à l'injonction d'intégration a poussé (et pousse encore) de nombreux parents à faire le choix unique du français, qui leur apparaît ainsi comme la clé de la réussite de leurs enfants à l'école et dans la société.

Il ne s'agit pas de juger ce choix, mais le problème est surtout qu'il se fait au détriment de l'autre (ou des autres) langue(s) familiale(s). Et j'ai eu l'occasion à plusieurs reprises d'entendre des interviewés exprimer le regret que leurs parents aient cédé à cette injonction de l'exclusivité du français, ou d'y avoir cédé eux-mêmes en tant que 
parents. Cela étant dit, il ne faut pas avoir une image trop binaire de ces phénomènes, puisque d'autres témoignages montrent qu'il est aussi possible de concilier investissement dans le français et transmission des langues familiales.

\section{Une multiplicité de pratiques}

Les pratiques de l'arabe maghrébin se déroulent aussi bien dans la sphère privée (famille, amis, proches...) que dans la sphère publique (médias, arts et spectacles ${ }^{(17)}$...). Cette séparation en deux sphères demeure assez schématique puisqu'elles se recoupent facilement, et notamment dans les pratiques que l'on rencontre sur Internet. Il existe en effet de nombreux sites utilisant, valorisant ou décrivant une variété d'arabe maghrébin. S'ils ne sont pas tous produits en France, ils sont en tout cas consultés ici. Ils sont issus d'individus ou d'associations dont la visée peut être informative, culturelle au sens large, ou communautaire ${ }^{(18)}$. Il n'est pas rare que les auteurs de ces sites y incluent un contenu linguistique (proverbes, lexiques, cours de langue...), que l'on retrouve aussi très souvent dans les commentaires (quand le site le permet) des internautes ${ }^{(19)}$. Ces mêmes internautes emploient l'arabe maghrébin sur les forums de discussion ou à travers les réseaux sociaux. Les locuteurs de France peuvent ainsi retrouver très facilement ceux du nord de l'Afrique.

À titre d'exemple, on peut citer : www.yabiladi.com, centré sur la culture marocaine ; la chaîne "télé derja" sur le site de partage de vidéos (www.youtube.com/ user/Telederja), qui propose des contenus en arabe algérien. Concernant les communautés juives, on peut en citer trois : www.dafina.net (juifs marocains) ${ }^{(20)}$, www.zlabia.com (juifs algériens) ${ }^{(21)}$ et www.harissa.com (juifs tunisiens) ${ }^{(22)}$.

Les sites concernant les pieds-noirs sont, comme ceux des juifs d'Afrique du Nord, souvent centrés sur l'histoire de la communauté avant les Indépendances, permettent aux membres de retrouver d'anciens amis ou voisins. Ils sont souvent constitués autour de lieux ou de moments ancrés dans le passé (les anciens du lycée français de Tunis), ou, pour les pieds-noirs d'Algérie, autour des anciens noms des villes (Philippeville, Orléansville...), par exemple : www.stozouit.free.fr/.Si on y retrouve peu de données linguistiques sur l'arabe, on y trouve des lexiques descriptifs du pataouète (français populaire avec des apports lexicaux de l'arabe, de l'italien, de l'espagnol, etc.). Quant aux sites consacrés aux harkis, on ne trouve pas cette dimension culturelle ou linguistique, les associations dont ils sont l'expression se situant plus dans une démarche politique de reconnaissance et de réparation (www.harki.net/article.php). Les pratiques sur Internet, hormis les supports audiovisuels, sont essentiellement écrites. Des pratiques écrites, certes, mais interactives. L'arabe maghrébin n'étant pas codifié à l'écrit, les locuteurs ont le choix entre la graphie arabe ou latine. C'est cette 
dernière qui apparaît le plus, pour une raison simple : les claviers d'ordinateurs, en France comme dans le nord de l'Afrique, sont en caractères latins.

D’une manière générale, les pratiques de l'arabe maghrébin sont multiples et, qu'il s'agisse de la sphère privée comme de la sphère publique, le mélange des langues est la règle ${ }^{(23)}$.Écartons toute idée de pureté ou d'évaluation de compétences linguistiques, les locuteurs mélangent naturellement les langues qu'ils ont à leur disposition : français, arabe maghrébin, berbère. Ce mélange va d'autant plus de soi que ces langues sont en contact depuis fort longtemps (près de deux siècles si l'on remonte à la colonisation de l'Algérie) en France comme dans le nord de l'Afrique. Les influences linguistiques réciproques sont nombreuses et en constante évolution. Ainsi, l'arabe maghrébin a une forte influence sur les parlers des jeunes, quelle que soit l'origine géographique ou sociale de leurs parents. Il faut également prendre en compte la multiplicité des répertoires, propre à tout locuteur de n'importe quelle langue, qui s'appréhende à différentes échelles sociales : famille, quartier, région, etc.

Même si l'on commence à avoir des indications intéressantes à travers certains travaux de recherche, on manque de données empiriques fines tout autant que d'éléments statistiques à grande échelle pour une meilleure prise en compte des pratiques effectives. Il y a ainsi comme un fossé entre la vivacité de l'arabe maghrébin que l'on perçoit très clairement dans la société française actuelle et le peu de place accordée à la diffusion et à la connaissance de cette langue par les institutions.

\section{Notes}

1. Le Conseil constitutionnel, se basant sur une lecture restrictive de l'article 2 de la Constitution, article ajouté en 1992 et qui stipule que "la langue de la République est le français", a jugé la charte contraire à la Constitution. La situation semble donc être bloquée et le fait que l'Assemblée nationale ait voté le 22 mai 2008, un amendement à l'article 1 de la Constitution ajoutant que les langues régionales appartiennent au patrimoine de la République, n'a pas fait avancer le débat.

2. Mon doctorat a notamment pour but de décrire qui sont les locuteurs de l'arabe maghrébin en France et quelles sont leurs pratiques langagières.

3. Pour une définition plus détaillée de l'arabe maghrébin, voir Caubet Dominique, "Larabe maghrébin", in Bernard Cerquiglini (dir.), Les Langues de France, Paris, Presses Universitaires de France, 2003, pp. 193-204.

4. Sauf indication contraire, je ne prendrai en compte que le territoire hexagonal.

5. Je n'entrerai pas ici dans la définition, ni dans la critique, de ces catégorisations.

6. Pour une analyse détaillée de ces indices, voir Caubet Dominique, "Immigrant languages and languages of France", in Monica Barni et Guus Extra (éds.), Mapping Linguistic Diversity in Multicultural Contexts, Berlin-New York, Mouton de Gruyter, 2008, pp. 163-193 ; Chaker Salem, "Berber, a 'long-forgotten' language of France", in Language and (Im migration in France, Latin America, and the United States: Sociolinguistic Perspectives, conference at the University of Texas at Austin, France-UT Institute for Interdisciplinary Studies, September 25-26 2003. http://www.utexas.edu/cola/insts/franceut/archives/Fall2003/ConfLangImmigration/chaker_english.pdf.

7. La situation de l'enseignement de l'arabe maghrébin est présentée dans Barontini Alexandrine, "Valorisation des langues vivantes en France : le cas de l'arabe maghrébin", in Le Français aujourd'hui, n 158, 2007, pp. 21-28.

8. Enquête menée à l'occasion du recensement de 1999 par l'Insee et l'Ined.

9. Deprez Christine, Filhon Alexandra et Heran François, "La dynamique des langues en France au fil du XX ${ }^{\text {ème }}$ siècle", in Population et sociétés, $\mathrm{n}^{\circ} 376$, Ined, 2002, p. 3.

10. Ibid., fig. 1, p. 2 . 
11. Caubet Dominique, "Langues et Musiques de France depuis les années quatre-vingt : pluralité linguistique et dimension populaire", in Carmen Alen Garabato et Henri Boyer (éd.), Les langues de France au XXI' siècle : vitalité sociolinguistique et dynamiques culturelles, Paris, L'Harmattan, 2007, pp. 51-75.

12. Lepoutre David et Cannoodt Isabelle, Souvenirs de familles immigrées, Paris, Odile Jacob, 2005, pp. 290-304.

13. Ayant grandi au Maroc mais dans un environnement plutôt francophone. Voir Barontini Alexandrine, "Pratiques de l'arabe maghrébin en France : trois familles (de tradition musulmane et juive) originaires du Maroc et d'Algérie", in Stephan Procházka and Veronika Ritt-Benminoun (éd.), Between the Atlantic and Indian Oceans. Studies on Contemporary Arabic Dialects. Proceedings of the 7th AIDA Conference, Neue Beihefte zur Wiener Zeitschrift für die Kunde des Morgenlandes, Berlin, Lit Verlag, 2008, pp. 67-79.

14. Née en France, de parents marocains.

15. Voir Barontini Alexandrine et Caubet Dominique, "La transmission de l'arabe maghrébin en France : état des lieux", Cahiers de l'Observatoire des pratiques linguistiques, "Migrations et plurilinguisme en France", n², Délégation générale à la langue française et aux langues de France, Éditions Didier, 2008, pp. 43-48.

16. J'entends par là celle qui considère le plurilinguisme comme source de problèmes et le monolinguisme comme la norme absolue. Ce point de vue, bien qu'en contradiction avec la réalité (le plurilinguisme est la règle) et avec de nombreux travaux de recherche, est toujours très prégnant en France, notamment dans le système scolaire.

17. Pour la musique, voir par exemple Caubet Dominique, "Langues et Musiques de France depuis les années quatre-vingt : pluralité linguistique et dimension populaire", op. cit.

18. J'emploie ici ce terme dans son sens premier, sans y mettre aucune connotation ni positive ni négative.

19. Voir Bensalah Amina, "Herméneutique du 'kif', valeur performative d'une expression idiomatique", in Michelle Auzanneau (dir.), avec la collaboration de Sarah Verdoia, La mise en cuvre des langues dans l'interaction, L'Harmattan, coll. Espaces discursifs, Paris, 2007, p. 101-119.

20. La dafina est le repas traditionnel du chabbat, à base de blé, pois chiches et viande cuit toute une nuit dans un four fermé à basse température. Le mot vient de la racine arabe "DFN" qui a trait à l'idée d'enterrement, d'étouffement.

21. La zlabia est un beignet tubulaire frit et trempé dans le miel.

22. La harissa est une purée de piment, dont la Tunisie est un des principaux producteurs.

23. Voir par exemple le travail de Jean-Michel Géa en Corse : Géa Jean-Michel, "Immigration et contacts de langues en Corse. L'exemple de deux familles marocaines", in Langage et Société, n 112, "Pratiques linguistiques en Corse", 2005 , pp. 57-78. 